\title{
Validity of Job Satisfaction Survey Scale in Chinese
}

\author{
LUAN Xiu-yun $^{1}$, ZHAI Qing-guo ${ }^{1,2}$, YANG Yu-wen ${ }^{3}$, WANG Zhi-hong ${ }^{4}$, ZHAI Yu-bo ${ }^{5}$ \\ 1 School of Business and Economics, Liaoning Shihua University, P. R. China, 113001 \\ 2 Department of Management, Monash University, Clayton, Vic. Australia, 3800 \\ 3 Research Center on Circular Economy, Nankai University, P. R. China, 300071 \\ 4 School of Business and Economics, Liaoning Technical University, P. R China, 125105 \\ 5 Faculty of Business and Economics, Melbourne University, Vic. Australia, 3010
}

\begin{abstract}
The aim of this study is to examine the factor structure of the scale of Job Satisfaction Survey (JSS), and its divergent and convergent validity in Chinese population. Data were collected with JSS from 1073 urban employees in Liaoning. Four alternative models were tested with confirmatory factor analysis. The first two models are models validated in the US, while the third and fourth models are composed of five commonly used dimensions of job satisfaction taken from JSS. The research found a poor model fit for the first two models, suggesting a possible national difference between China and the US. However, model three and model four displayed a good model fit, suggesting that the five dimensions in JSS (satisfaction with nature of work, with supervision, with co-worker, with promotion, and with pay) are five distinct dimensions. The correlations between these five facet job satisfaction and PA and NA demonstrated convergent and divergent validity of the scales for these five dimensions of job satisfaction.
\end{abstract}

Keywords: China, confirmatory factor analysis, job satisfaction survey, scale, validity

\section{Introduction}

Job satisfaction is one of the most important organizational variables, as research has found that it is linked with various work-related outcomes, such as job performance ${ }^{[1,2]}$, organizational citizenship behaviour ${ }^{[3,4]}$ and turnover intentions ${ }^{[5-7]}$, Locke ${ }^{[8]}$ defined job satisfaction as "a pleasurable or positive emotional state resulting from the appraisal of one's job or job experience". Job satisfaction can be about job as a whole or about the facets of a job such as relationship with other people in the work place, rewards from work and nature of work etc. ${ }^{[9]}$. Researchers in the west have designed several reliable and valid multi-item measures to measure dimensions of job satisfaction [10, 11] However, when dimensions of job satisfaction are studied in China, researchers often use single-item indicator ${ }^{[12-14]}$ or self-designed survey instruments ${ }^{[15,16]}$. The use of single item measures has been particularly criticized on two grounds. First, a single-item indicator is typically less reliable than a multiple item measure. For example, Andrews and Whithey ${ }^{[17]}$ found single item indicator of personal well-being to have relatively low reliability (test-retest correlation .40- .66), even when respondents were asked twice only one hour apart. The test-retest correlations for a multi-item personal wellbeing scale were much higher, ranging from .82 to .84 . Second, a single indicator cannot capture the multiple dimensions or facets of complex constructs such as job satisfaction ${ }^{[18]}$. In terms of the self-developed measures in Chinese studies, the main limitation of self-developed measures is that their psychometric property is not strictly tested.

The aim of this study is to test the factor structure of Job Satisfaction Survey (JSS), a well-established survey instrument in the US in a Chinese context. Although, the validity and reliability of JSS have been proved in previous studies in the US ${ }^{[19]}$, the validity and reliability of the full 36 item JSS scale has not been tested in China. Sekaran ${ }^{[20]}$ recommended to investigate into the crosscultural stability of the job satisfaction constructs before applying job satisfaction scales developed in the US to other cultural settings. Because of the differences in cultural values and language between the US and China ${ }^{[21]}$, the translation of an English measurement may not always be suitable for population speaking another language, such as Chinese ${ }^{[22,23]}$. According to Lamond, Spector, Mcdonald, $\mathrm{Wu}$ and Hosking ${ }^{[24]}$, individuals in different countries would view some items differently. Furthermore, language translation may result in discrepancies between the original and the target survey that can affect equivalence of the meaning of the constructs, and some items may be inappropriate for the target culture or may be badly translated into the target language ${ }^{[25]}$. Empirical studies ${ }^{[26]}$ have demonstrated potential cross-cultural differences in the underlying factor structure of job satisfaction. To summarize, the main contribution of the current study is to test whether the scale of JSS is valid in a specific non-western context, for translation and cultural differences may make scale developed in the west inappropriate for use in China.

The remainder of the article is set out as follows. The next section discusses the characteristics of the 
sample, the survey instrument, the method for CFA, and the method to test the divergent and convergent validity of the scale. Section three is the result. The discussion section is given in section four. The final section is the conclusion.

\section{Method}

\subsection{The sample and procedure}

A convenience sampling method was used for data collection. Data was collected from Dalian, Fushun and Shenyang in 2008. The sample covered a wide range of industry groups, including manufacturing, health services, education, and government branches. The number of completed surveys was 1073. The characteristics of respondents are reported in Tab. 1.

Tab. 1 demonstrates that the sample is composed of a little more females than males. Most of the respondents are young, with 90 percent aged less than 45 years old. More than half of the respondents have four years or more of higher education.

\subsection{Measures}

Job satisfaction was measured with the 36 -item JSS scale. The response format is a six point-Likert scale from $1=$ disagree very much to $6=$ agree very much. Positive and negative affectivity was measured with a 10 -item short form PANAS scale ${ }^{[27]}$. As the original scale is in English, translation and back-translation is used for the translation of the scale.

Tab.1 Profile of the survey respondents

\begin{tabular}{lll}
\hline Variables & $\mathrm{N}$ & $\%$ \\
\hline Gender & & \\
Male & 462 & 43.4 \\
Female & 602 & 56.6 \\
Age (years) & & \\
$<25$ & 182 & 17.1 \\
$26-30$ & 333 & 31.4 \\
$31-35$ & 162 & 15.3 \\
$36-40$ & 165 & 15.5 \\
$41-45$ & 119 & 11.2 \\
$46-50$ & 54 & 5.1 \\
$51-55$ & 32 & 3.0 \\
$56-60$ & 15 & 1.4 \\
Marital status & & \\
Single & 376 & 35.3 \\
Married & 688 & 64.7 \\
Education & & \\
Junior secondary & 45 & 4.3 \\
Senior secondary & 125 & 11.8 \\
Polytechnic & 293 & 27.7 \\
Bachelor & 492 & 46.5 \\
Master or PHD & 103 & 9.7 \\
Income & & \\
Less than 500 & 15 & 1.4 \\
501-1000 & 106 & 10.0 \\
1001-1500 & 202 & 19.0 \\
1501-2000 & 251 & 23.6 \\
2001-5000 & 445 & 41.8 \\
Over 5000 & 46 & 4.3 \\
\hline & &
\end{tabular}

One of the authors translated the original English version into Chinese and subsequently a second bilingual speaker back-translated it into English. The backtranslation and the original were compared, and discrepancies between those two versions were resolved through discussion between translators to ensure equivalence of both Chinese and English versions. The descriptive statistics of the measures are shown in Tab. 2.

Tab 2 demonstrated that two sub-scales in job satisfaction, satisfaction with co-works, satisfaction with operating procedures had a Cronbach's alpha lower than .60 , which is the hurdle for cross-cultural studies ${ }^{[28]}$ The low reliabilities of two of the sub-dimensions of job satisfaction suggest that this full 36-item JSS scale may not be suitable for Chinese context.

\subsection{Data analysis method}

Confirmatory factor analysis (CFA) was used to test the factor structure of JSS. Four alternative models are proposed in this study. Model one is a nine dimension model of job satisfaction: satisfaction with pay, promotion, fringe benefits, contingent rewards (appreciation and recognition), supervision, co-workers, nature of work, communication and operating procedures The nine dimensional model was proposed by Spector ${ }^{[10]}$. Each dimension is measured with four items. The nine dimensional model has been proved to be a reliable and valid measure of job satisfaction in the United States ${ }^{[19]}$. In model two, there are four dimensions: satisfaction with agents, with rewards, with content, and with context. According to Locke ${ }^{[8]}$, job satisfaction could be categorized into these four broad categories. The 36 items in JSS are organized in these four dimensions. The agent factor is consisted of the dimension of supervision, and co-workers in JSS. The rewards factor is composed of the dimension of pay, promotion, contingent rewards, and fringe benefit in JSS. The content factor is the dimension of nature of work in JSS. The context factor is composed of the dimension of operating procedures, and communication in JSS. Empirical studies in the US ${ }^{[29]}$ supported the four dimensional model.

Tab.2 Descriptive statistics and Cronbach's alphas of the measures

\begin{tabular}{llll}
\multicolumn{4}{c}{ of the measures } \\
\hline Sub-scale & means & s.d. & Alpha \\
\hline PA & 3.46 & 0.62 & .72 \\
NA & 2.43 & 0.63 & .75 \\
Supervision & 16.65 & 3.71 & .80 \\
Nature of work & 16.08 & 3.59 & .74 \\
Pay & 13.19 & 3.44 & .68 \\
Promotion & 13.34 & 3.21 & .65 \\
Co-workers & 16.93 & 2.61 & .50 \\
Benefit & 13.20 & 3.79 & .76 \\
Rewards & 14.58 & 3.07 & .61 \\
Operating procedures & 13.41 & 2.76 & .41 \\
Communication & 15.45 & 3.03 & .59 \\
\hline
\end{tabular}


Tab.3 Comparison of alternative CFA models of the JSS

\begin{tabular}{llllllllll}
\hline Model & $\chi^{2}$ & DF & $\chi^{2} / \mathrm{DF}$ & P & CFI & RMSEA & LO90 & HI90 \\
\hline Model 1 & 3027 & 558 & 5.42 & .000 & .79 & .07 & .06 & .07 \\
Model 2 & 3496 & 558 & 5.95 & .000 & .76 & .07 & .07 & .07 \\
Model 3 & 885 & 160 & 5.54 & .000 & .87 & .07 & .06 & .07 \\
Model 4 & 683 & 125 & 5.46 & .000 & .90 & .07 & .06 & .07 \\
\hline
\end{tabular}

Model three includes five components in JSS: satisfaction with pay, with supervision, with co-worker, with promotion, and with nature of work. These five dimensions are the original facet job satisfactions in JSS. According to Spector ${ }^{[19]}$, these five facets are the most commonly studied dimensions in job satisfaction research. Model four is the model in which all the items with a factor loading of less than .40 are dropped from model three.

According to Harris and Schaubroeck ${ }^{[30]}, \mathrm{CFA}$ is most appropriate for use with established measures. Following Hair, Black, Babin, Anderson and Tatbam ${ }^{[31]}$, multiple fit indices are used to assess the model's goodness-of-fit. The chi-square, degrees of freedom (df), comparative fit index (CFI), and root-mean-square error of approximation (RMSEA) are reported. Byrne [32] suggested a CFI of no less than .90, and a RMSEA of no more than .08 for a good model fit. In terms of factor loadings, a factor loading of .40 or above was used as the criterion for keeping an item in a scale because it is commonly considered "significant" in defining a factor [31].

Correlations between the facet job satisfactions and positive affectivity (PA) and negative affectivity (NA) was used to test the convergent and divergent validity of the measures of facet job satisfaction. First, according to Bowling, Hendricks and Wagner ${ }^{[33]}$, the facet job satisfaction should be positively related to positive affectivity (PA) because high PA individuals are more likely to be in objectively more favorable situations, and tends to recall and interpret their work situations more favorably. On the contrary, individuals high in negative affectivity (NA) should be negatively related to the facet job satisfaction because high NA individuals are likely to be in objectively unfavorable situations and recall and perceive their work situations negatively.

According to Bowling et al. ${ }^{[33]}$, the relationship between satisfaction with pay, satisfaction with promotion and PA should be stronger than the relationship between these two facet job satisfaction and $\mathrm{NA}$, as PA is responsible for one's sensitivity with rewards, while NA is responsible for one's sensitivity with punishment, and pay and promotion represent two important work rewards. In terms of satisfaction with supervisors and with co-workers, their relationship with NA should be stronger than their relationship with PA, as NA are more likely to have a stronger impact on interpersonal relationships than $\mathrm{PA}{ }^{[33]}$. This expectation between the facet job satisfaction and PA and NA is assessed by examining the correlations of the two affectivity variables with the dimensions of job satisfaction.

\section{Results}

The CFA results of the model fit indices are shown in Tab. 3.

As shown in Tab. 3, the model fit indices CFI of the first two models are well below the hurdle of .90 for good model fit. The value of model fit index CFI of .87 in model three is close to good model fit. The index of RMSEA in model three is .07, which falls in the range for good model fit of RMSEA less than .08. After deletion of two items with factor loadings less than .40 in model three, the model fit index CFI of model four increases to .90. Importantly, the RMSEA of .08 falls in the range of good model fit demonstrating good model fit

The factor loadings of the items in model three are demonstrated in Tab. 4.

Tab.4 demonstrates that except two items in the dimension of satisfaction with co-worker, all the items have factor loadings larger than .40. The results indicate that all the items except two loaded decently on the intended dimensions of job satisfaction.

The correlations between the five dimensions of job satisfaction and PA, NA are displayed in Tab. 5.

Tab.5 demonstrated that PA is statistically significantly positively related with all the five dimensions of job satisfaction, while NA is statistically significantly negatively related with four of the five dimensions of job satisfaction. The relationships between satisfaction with supervisors, with co-workers and NA are stronger than the relationship between these two facet job satisfaction and PA. The relationship between satisfaction with pay, with promotion and PA are stronger than the relationship between these two facet job satisfaction and NA. All these correlations demonstrated convergent and divergent validity of the measures of these five dimensions of job satisfaction.

\section{Discussion}

The research found a poor model fit for both the nine factor model and the four factor model, suggesting that the factor structure of Chinese people's job satisfaction may be different from those in the US. The finding is consistent with findings in previous studies in Singapore ${ }^{[26]}$, and in India ${ }^{[34]}$, where factor structures were also found to be different from those in the US. Furthermore, the Cronbach's alphas of two of the nine sub-scales were less than .60, indicating low internal 
Tab.4 Factor loading of the items from model three

\begin{tabular}{|c|c|c|c|c|c|}
\hline Item & PY & PM & SV & $\mathrm{CW}$ & WK \\
\hline I feel satisfied with my chances for salary increases. & .69 & & & & \\
\hline I feel unappreciated by the organization when I think about what they pay me. (R) & .53 & & & & \\
\hline Raises are too few and far between. (R) & .50 & & & & \\
\hline I feel I am being paid a fair amount for the work I do. & .55 & & & & \\
\hline I am satisfied with my chances for promotion. & & .67 & & & \\
\hline People get ahead as fast here as they do in other places. & & .66 & & & \\
\hline Those who do well on the job stand a fair chance of being promoted. & & .55 & & & \\
\hline There is really too little chance for promotion on my job. (R) & & .40 & & & \\
\hline I like my supervisor. & & & .79 & & \\
\hline My supervisor shows too little interest in the feelings of subordinates. (R) & & & .70 & & \\
\hline My supervisor is unfair to me. $(\mathrm{R})$ & & & .71 & & \\
\hline My supervisor is quite competent in doing his/her job. & & & .69 & & \\
\hline There is too much bickering and fighting at work. (R) & & & & .34 & \\
\hline I enjoy my coworkers. & & & & .53 & \\
\hline $\begin{array}{l}\text { I find I have to work harder at my job because of the incompetence of people I work } \\
\text { with. (R) }\end{array}$ & & & & .27 & \\
\hline I like the people I work with. & & & & 68 & \\
\hline My job is enjoyable. & & & & & .77 \\
\hline I feel a sense of pride in doing my job. & & & & & .69 \\
\hline I like doing the things I do at work. & & & & & .72 \\
\hline I sometimes feel my job is meaningless. $(\mathrm{R})$ & & & & & .56 \\
\hline
\end{tabular}

Note: Bold indicates that the factor loading is over .40. $\mathrm{R}=$ reversed item. PY: satisfaction with pay; PM: satisfaction with promotion; SV: satisfaction with supervision; CW: satisfaction with coworkers; WK: satisfaction with nature of work.

Tab.5 Correlations of the five dimensions of job satisfaction and PA and NA

\begin{tabular}{lcc}
\multicolumn{3}{c}{ of job satisfaction and PA and NA } \\
\hline Satisfaction with & PA & NA \\
\hline Nature of Work & .34 & -.21 \\
Pay & .15 & -.13 \\
Promotion & .12 & -.05 \\
Supervision & .16 & -.19 \\
Coworkers & .20 & -.25 \\
\hline
\end{tabular}

$p<.01$ for $r>.12 ; p<.05$ for $r>.11$

consistency for those sub-scales. The results indicate that there may be cultural differences on the structure of job satisfaction across nations.

The explanation for the finding of the unsuitability of the nine dimensionality job satisfaction measure for Chinese may be that because of the differences between China and America in culture, language, and economic development, conceptually the dimensionality of Chinese is different from Americans. For instance, satisfaction with pay, with fringe benefits and with contingent rewards are three separate dimensions for American, however Chinese do not distinguish them. Specifically, when the researchers explored the factor structure of the data with principal component analysis with varimax rotation, eight components with eigenvalues larger than 1 were extracted. Most of the items in the dimensions of pay, fringe benefit and contingent rewards fall into one factor, rather than three separate factors. When Chinese measures of job satisfaction are developed, pay and benefit are also often used as one facet, rather than two separate facets ${ }^{[35,36]}$.

The CFA results demonstrated good model fit for the five dimensions of job satisfaction: satisfaction with pay, with promotion, with supervision, with co-workers, and with nature of work. The findings suggest that the five sub-scales of JSS were separate from each other. Except two items in the subscale of satisfaction with coworkers, all the item factor loadings are above .40. The item "I find I have to work harder at my job because of the incompetence of people I work with" also had a factor loading of less than .40. This item could be measuring the interrelationship between people's work, rather than the interpersonal relationship. So this item may not be fit in Chinese culture. The item "There is too much bickering and fighting at work" had a low factor loading. The low factor loading could be related with Chinese culture. Chinese are reluctant to express strong feelings in the work place especially negative feelings towards other people.

The explanation for the applicability of the five subdimensions of job satisfaction could be that Chinese are familiar with these dimensions and they are conceptually different constructs. For instance, the role of supervisors and co-worker could be quite different in the decision of pay raise, promotion, provision of training opportunities for an individual. The different role of supervisor and coworkers in one's career growth and getting rewards could make satisfaction with supervisor and co-worker as distinct constructs for Chinese. When Chinese measures of job satisfaction are developed, similar components such as these five are also found ${ }^{[35,36]}$.

The finding of a positive relationship between the five facet job satisfaction and PA, and a negative relationship between four facet job satisfaction and NA are consistent with findings in previous meta-analyses ${ }^{[33,}$ ${ }^{37]}$. This suggested that all the facet job satisfaction and PA and NA relationships are in the expected directions. The finding of a stronger relationship between satisfaction with supervisor, with co-worker and NA than 
the relationship between these two facet job satisfaction and PA is also consistent with previous meta-analyses ${ }^{[33 \text {, }}$ 37]. The research also found a stronger relationship between satisfaction with promotion, with pay and PA than the relationship between these two facet job satisfaction and NA. All these are consistent with previous meta-analyses ${ }^{[33,37]}$. These findings suggest the divergent and convergent validity of these five facet job satisfaction.

Of all the alphas of the five dimensions of job satisfaction, alpha of satisfaction with co-workers is the lowest in current study, which is similar to the findings in other studies, where alpha of this dimension was also found to be one of the bottom two among the five dimensions ${ }^{[10,26,34]}$. After deletion of the two items with factor loading less than .40 in the dimension of satisfaction with co-workers, the Cronbach's alpha of satisfaction with co-workers increased to .60. All these suggest that the scales for the five dimensions of job satisfaction could be used for Chinese context after some minor modification on the scale of satisfaction with coworker.

\section{Conclusion}

The aim of this study is to determine the crosscultural suitability of JSS in Chinese employees. The research found that overall the nine dimension model demonstrated poor construct validity, suggesting that the nine dimensions of JSS is not generalizable to Chinese employees. CFA result, the relationship between the five dimensions of job satisfaction and PA and NA, and the reliability of the scales demonstrated that the five subdimensions of satisfaction with nature of work, with supervisor, with co-worker, with pay, and with promotion in JSS can be used to measure job satisfaction in Chinese setting with some minor modification on the dimension of satisfaction with co-workers.

\section{References}

[1]Hui, T. An empirical research on the relationship between job satisfaction and performance of $R$ \& $D$ workers. Science of Science and Management of S \& T, 2006(5): 145-148. (in Chinese)

[2]Judge, T.A., et al. The job satisfaction- job performance relationship: A qualitative and quantitative review. Psychological Bulletin, 2001, 127(3):376-407.

[3]Wang, X. and H. Sun, Empirical study of OCB in China. China Economist, 2005(2): 265-266. (in Chinese) [4]Meyer, J.P., et al. Affective, continuance, and normative commitment to the organization: A metaanalysis of antecedents, correlates, and consequces. Journal of Vocational Behavior, 2002, 61:20-52.

[5]Chen, Y. and Z. Li. The relationship between career plateaus, job satisfaction, organizational commitment and turnover intentions. Science and Technology Management Research, 2009(12): 437-440. (in Chinese)
[6]Zhang, Z. and S. Zhao. Empirical study on the determinants of intellectuals turnover to other regions: A study on the north Jiangshu. Management World, 2007(8): 95-103. (in Chinese)

[7]Price, J.L. Reflections on the determinants of voluntary turnover. International Journal of Manpower, 2001, 22(7/8): 600-624.

[8]Locke, E.A. The nature and causes of job satisfaction. in Handbook of Industrial and Organizational Psychology, M.D. Dunnette, Editor. Rand Mcnally: Chicago, 1976:1297-1349.

[9]Fields, D.L. Taking the measure of work- A guide to validated scales for organizational research and diagnosis. Thousand Oaks: Sage, 2002.

[10]Spector, P.E. Measurement of human service staff satisfaction: Development of the job satisfaction survey. American Journal of Community Psychology, 1985, 13: 693-713.

[11]Smith, P.C., L.M. Kendall, and C.L. Hulin. The measurement of satisfaction in work and retirement. Chicago IL: Rand McNally, 1969.

[12]Hu, P. and J.a. Chen. Empirical study on job satisfaction of intellectuals. Management of Scientific Research, 2003(4):139-144. (in Chinese)

[13]Zhu, M., X. Wu, and L. Feng. Empirical study on the incentives of employees in different ownership. Finance and Economics, 2001(4):44-49. (in Chinese)

[14]Zhang, M. and S. Li. Empirical study of the determinants of employee job satisfaction. Statistical Research, 2001(8):33-37. (in Chinese)

[15]Liu, Y., H. Yang, and Y. Zhao. Empirical study of employee job satisfaction. Modern Management Science, 2006(5): 5-7. (in Chinese)

[16]Liu, F. and J. Zhang. Validity of the survey instrument of employee job satisfaction and research on the determinants of employee job satisfaction in private owned enterprises. Nankai Economic Review, 2004, 7(3): 97-104. (in Chinese)

[17]Andrews, F.M. and S.B. Whithey. Social indicators of well-being: Americans' perception of life satisfaction. New York: Plenum, 1976.

[18]Krueger, A.B. and D.A. Schkade. The reliability of subjective well-being measures. National Bureau of Economic Research working papers 13027, 2007.

[19]Spector, P.E. Job satisfaction, application, assessment. Causes, and Consequences. Sage publication, Inc, 1997.

[20]Sekaran, U. Methodological and theoretical issues and advancements in cross-cultural research. Journal of International Business Studies, 1983, 14(2): 61-73.

[21]Hofstede, G. Cultures and Organizations. New York: McGraw-Hill, 1991.

[22]Andolssek, D.M. and J. Sstebe. Multinational perspectives on work values and commitment. International Journal of Cross Cultural Management, 2004, 4:181-209.

[23]Tayyeb, S. and M.N. Riaz. Validation of the threecomponent model of organizational commitment in 
Pakistan. Pakistan Journal of Psychological Research, 2004, 19:123-149.

[24]Lamond, D., et al. Of emics and etics: The development of a cross-cultural facet measure of job satisfaction. in Academy of Management Proceedings \& Membership Directory: Academy of Management, 2001.

[25]Cong, L., I. Borg, and P.E. Spector. Measurement equivalence of the German job satisfaction survey used in a multinational organization: Implications of Schwartz's culture model. Journal of Applied Psychology, 2004, 89(6): 1070-1082.

[26]Spector, P.E. and J. Wimalasiri. A cross-cultural comparison of job satisfaction dimensions in the United States and Singapore. International Review of Applied Psychology, 1986, 35: 147-158.

[27]Thompson, E.R. Development and validation of an internationally reliable short-form of the positive and negative affect schedule (PANAS). Journal of Crosscultural Psychology, 2007, 38(2): 227-242.

[28]Sekaran, U. Research methods for business: A skillbuilding approach. 2 ed. New York: John Wiley and Sons, Inc, 1992.

[29]Michaels, C.E.J. The dimensionality of job satisfaction. University of South Florida: United States Florida, 1983.

[30]Harris, M.M. and J. Schaubroeck. Confirmatory modeling in organizational behavior/human resource management: Issues and applications. Journal of Management, 1990, 16: 337-360.

[31]Hair, J.F., et al. Multivariate data analysis. 6th ed. New Jersey: Pearson Prentice Hall, 2006.

[32]Byrne, B.M. Structural equation modeling with AMOS: Basic concepts, applications, and programming. Mahwah, New Jersey: Lawrence Erlbaum Associates, 2001.

[33]Bowling, N., E. Hendricks, and S. Wagner. Positive and negative affectivity and facet satisfaction: A metaanalysis. Journal of Business \& Psychology, 2008, 23(3/4): 115-125.

[34]Takalkar, P. and M.D. Coovert. International replication note: The dimensionality of job satisfaction in India. Applied Psychology: An International Review, 1994, 43(3): 415-426.

[35]Lu Jia, Shi Kan, Yang Jifeng. Survey scale and method of job satisfaction. Human resource development of China, 2001,(1):15-17. (in Chinese)

[36]Tao Hong, Hu Jing-chao, Wang Lin, Liu Xiao-hong. Development of a scale for job satisfaction assessment in nurses. Academic Journal of Second Military Medical University, 2009,30(11): 1292-1296.(in Chinese)

[37]Ng, T.W.H. and K.L. Sorensen. Dispositional affectivity and work-related outcomes: A meta-analysis. Journal of Applied Social Psychology, 2009, 39(6): 1255-1287. 